Proc. Estonian Acad. Sci. Eng., 2005, 11, 3, 245-260

\title{
Profiles of waves from high-speed ferries in the coastal area of Tallinn Bay
}

\author{
Tarmo Soomere $^{\mathrm{a}}$, Reio Põder ${ }^{\mathrm{a}}$, Kalev Rannat ${ }^{\mathrm{a}}$ and Andres Kask ${ }^{\mathrm{b}}$ \\ ${ }^{a}$ Institute of Cybernetics, Tallinn University of Technology, Akadeemia tee 21, 12618 Tallinn, \\ Estonia; soomere@cs.ioc.ee \\ b Marine Systems Institute, Tallinn University of Technology, Akadeemia tee 21, 12618 Tallinn, \\ Estonia
}

Received 15 October 2004, in revised form 18 April 2005

\begin{abstract}
The shape and properties of long ship-generated waves approaching shallow coastal areas of Tallinn Bay are studied based on recordings of water surface time series. For typical leading wake waves non-linear effects become significant at depths of 10-15 m. A large part of waves (with the height over $0.4 \mathrm{~m}$ ) have the shape of cnoidal waves in shallow areas with depths of 4-5 m. The shapes of the largest wake waves are close to the solitary wave solutions of the Korteweg-de Vries equation. Such waves excite considerably larger velocities of water particles than sinusoidal waves of equal height and length.
\end{abstract}

Key words: fast ferries, ship wash, cnoidal waves, wave measurements.

\section{INTRODUCTION}

The importance of the contribution of the ship traffic to the local hydrodynamic activity in inland waterways and narrow straits has been recognized for a long time. Ship wakes can cause an extensive resuspension of bottom sediments and essentially contribute to the shoreline erosion $\left[^{1-3}\right]$, trigger ecological disturbance and cause harm to the aquatic wildlife $\left.{ }^{4,5}\right]$. They may serve as a qualitatively new forcing component of the aquatic ecosystem in certain open sea areas with low natural wave activity, in particular, in non-tidal areas that are sheltered from long waves $\left[{ }^{6,7}\right]$. The central concern is the possible impact of direct mechanical disturbances of bottom sediments $\left[{ }^{8,9}\right]$ that may lead to a potential intensification of sediment transport and beach destruction processes $\left[{ }^{10,11}\right]$ and to an overall decrease of water quality in areas, affected by ship waves $\left[{ }^{12}\right]$.

The excessive influence of wakes of high-speed ships occurs when wake waves are much longer than wind waves $\left[{ }^{13,14}\right]$. For example, typical wave 
periods in Tallinn Bay are 2-4 s and rarely reach $6-7 \mathrm{~s}\left[{ }^{15}\right]$. The leading wake waves frequently have a height of about $1 \mathrm{~m}$ and a period of $10-15 \mathrm{~s}\left[{ }^{14}\right]$. Such waves extremely seldom occur in natural conditions in certain regions of semienclosed seas. They are qualitatively similar to long-period ocean swell. Together with wind waves, they may form bi-modal wave systems, impact of which on various coastal processes may be much higher compared with that of wave systems with a single spectral peak and a comparable total energy $\left[{ }^{16,17}\right]$.

The properties and influence of ship waves usually have been calculated with the use of the assumption that they can be described by the classical linear wave theory $\left[^{8,10,12}\right]$. The length of leading waves of wakes from high-speed ships exceeds $100 \mathrm{~m}$ in areas with a depth of less than $10 \mathrm{~m}$. At smaller depths, such waves with a height of about $1 \mathrm{~m}$ cannot be considered as linear ones and even higher-order classical theories, for instance the Stokes wave theory $\left[{ }^{18}\right]$, are not always applicable. In this context, it is somewhat amusing that despite of major advances in fluid mechanics and naval architecture during the last decades, the classical, basically linear, Kelvin theory of steady wake (optionally with minor corrections) is still very much used for the description of vessel wakes also in the trans- and supercritical regime $\left[{ }^{10}\right]$, where apparently no steady regime exists neither in channels $\left[{ }^{19,20}\right]$ nor in unbounded sea areas $\left[{ }^{21,22}\right]$.

Overall progress in computational fluid mechanics has considerably increased the knowledge in dynamic description of vessel wakes. A general limitation of existing models is that today neither of them is able to correctly reproduce the flow simultaneously near the vessel and in the far field. According to $\left[^{6}\right]$, the Boussinesq-type models $\left[{ }^{23-27}\right]$, based on weakly non-linear description of surface waves, usually give more realistic results in shallow water than spectral models $\left[{ }^{13,28}\right]$ that rely on the basically linear wave behaviour. The common opinion is that, owing to large variability of wave-making and local conditions, regardless of what type of numerical model is the most appropriate, it is necessary to validate it, based on in situ measurements, before it can be used for managing wake wash in a particular situation $\left.{ }^{6,10}\right]$. Thus the choice of a reasonable wave model is an important step in the prediction of wake wash and its influence.

An appropriate model for the description of ship waves in coastal areas (that basically are long finite-amplitude surface waves in shallow water) is the Korteweg-de Vries $(\mathrm{KdV})$ equation $\left[{ }^{18}\right]$. Its periodic solutions are called cnoidal waves, because they can be explicitly expressed in terms of the so-called cnoidal (Jacobi elliptic) functions. Cnoidal waves have more narrow crests and more broad troughs than sine waves. The long-wave limit of a cnoidal wave is the KdV soliton. A moving disturbance in open sea areas seldom forms solitary waves of considerable height $\left[{ }^{21,29,30}\right]$. Yet long waves that approach shallow regions may excite sequences of highly non-linear or even soliton-like structures $\left[{ }^{31}\right]$. Recently it has been shown that certain dramatic effects may occur owing to non-linear interactions between two trains of soliton-like waves in the framework of the Kadomtsev-Petviashvili equation [ $\left.{ }^{32,33}\right]$. 
Theoretically, the shape and properties of long waves with appropriate parameters, propagating in a shallow region with an ideal flat bottom, should match those of the corresponding solution of the KdV equation. Although experimental evidence $\left[{ }^{10,14,29}\right]$ indicates that the shape of ship waves in shallow water is not sinusoidal, the sea bottom is never perfect and in realistic conditions it is not a priori clear what exactly happens when waves approach coastal areas that have two-dimensional bottom with many imperfections. The coastal area of the Tallinn Bay is an example of a region with very complex bathymetry adjacent to a ship lane, hosting extremely intense traffic of fast ferries.

The shape of the waves is extremely important, because many properties of water particles in long linear and weakly non-linear waves (in particular, velocity components) depend linearly on the surface displacement. The purpose of the current paper is to study the actual appearance of long ship waves, approaching shallow coastal areas. Based on the water surface time series, it is shown that a large part of waves from fast ferries in the coastal area of Tallinn Bay match the shape of cnoidal waves. For a wave with given height and length, the cnoidal wave theory predicts considerably larger velocities of water particles than the linear theory. For typical ship waves in the coastal zone of Tallinn Bay the difference may be a few times. Therefore, the possible adverse influence of long ship-generated waves may be much larger than its estimates on the basis of the classical wave theory.

\section{LINEAR AND CNOIDAL WAVES}

The shape of water surface (wave profile) in a plane linear (sine) wave is $\zeta(x, t)=a \cos (\kappa x-\omega t)$, where $a$ is the wave amplitude, $H=2 a$ is the wave height, $\kappa=2 \pi / L$ is the wave number (the length of the wave vector), $L$ is the wavelength, $x$ and $t$ are spatial and time coordinates, respectively, $\omega=\sqrt{g \kappa \tanh \kappa h}$ is the angular frequency, $T=2 \pi / \omega$ is the wave period, $h$ is the calm water depth and $g$ is acceleration due to gravity $\left[{ }^{18}\right]$. If the $z$ axis is directed upwards and $z=0$ is the calm water surface, the horizontal $u(x, z, t)$ and vertical $w(x, z, t)$ components of the velocity of water particles at the depth $z$ are:

$$
u=\frac{a g \kappa}{\omega} \frac{\cosh \kappa(z+h)}{\cosh \kappa h} \cos (\kappa x-\omega t), \quad w=\frac{a g \kappa}{\omega} \frac{\sinh \kappa(z+h)}{\cosh \kappa h} \sin (\kappa x-\omega t) .
$$

Linear wave theory has been and currently is widely used for the description of surface waves and their interactions, provided the wave height is small compared to the wave length and water depth. The formal basic requirement is $\kappa H / 2<<1\left[{ }^{18}\right]$. The period of a typical wave from a fast ferry in the Tallinn Bay area is about $10 \mathrm{~s}\left[{ }^{14}\right]$. Its length is $L \geq 30 \sqrt{h} \mathrm{~m}$; thus water with depth of $h \leq 10 \mathrm{~m}$ can be already considered as shallow. An appropriate non-dimensional parameter in shallow areas (where the wave height is an appreciable fraction of 
the water depth) is the Ursell number $U=H L^{2} h^{-3}\left[{ }^{18}\right]$. When $U \approx 1$, the linear theory is useful in many aspects, even when the condition $\kappa H / 2<<1$ is violated. For moderate Ursell numbers (up to $U \approx 75$ ) and $L / h<10$, various modifications of Stokes wave theory can be used $\left[{ }^{18}\right]$. The Ursell number for such a wave in the coastal area $(h \approx 3 \mathrm{~m})$ is $U \approx 100 H$ and already ship waves of moderate height $(H \approx 0.5 \mathrm{~m})$ correspond to $U \approx 50$. For even longer or higher waves (that frequently occur in the area in question $\left.\left[{ }^{14}\right]\right)$, or for smaller depths, the Stokes wave theory is generally incorrect $\left[{ }^{18}\right]$ and the cnoidal wave theory is preferable.

For simplicity, in this paper we consider only first-order cnoidal waves, for which the term $O\left(\kappa^{2} h^{2}\right)$ in the dispersion relation $\omega=\kappa \sqrt{g h}\left(1-\frac{1}{6} \kappa^{2} h^{2}\right)$ for the Korteweg-de Vries equation is neglected. The shape $\zeta(x, t)$ of cnoidal waves (Fig. 1a) and the horizontal and vertical velocity of water particles in such a wave at a depth $z$ are $\left[{ }^{18}\right]$ :

$$
\begin{gathered}
\zeta(x, t)=h_{t}-h+H \mathrm{cn}^{2}(\theta ; m), \\
\frac{u(x, z, t)}{\sqrt{g h_{t}}}=\left[m \mathrm{cn}^{2}(\theta ; m)-m+\frac{1}{2}\right] \frac{H}{m h_{t}}-1, \quad \alpha=\sqrt{\frac{3 H}{4 m h_{t}}}, \\
\frac{w(x, z, t)}{\sqrt{g h_{t}}}=2 \alpha H \frac{z+h}{h_{t}^{2}} \operatorname{cn}(\theta ; m) \operatorname{sn}(\theta ; m) \operatorname{dn}(\theta ; m), \\
\theta=\frac{\alpha L}{h_{t}}\left(\frac{x}{L}-\frac{t}{T}\right)=2 K(m)\left(\frac{x}{L}-\frac{t}{T}\right),
\end{gathered}
$$

where $h_{t}=h+H m^{-1}\left[1-m-E(m) K^{-1}(m)\right]$ is the minimum water depth at the wave trough, $K(m)$ and $E(m)$ are the complete elliptic integrals of the first and the second kind, respectively, and $\operatorname{cn}(\theta ; m), \operatorname{sn}(\theta ; m)$ and $\operatorname{dn}(\theta ; m)$ are Jacobi elliptic functions with the parameter $m$ (Fig. 1, frequently another parameter $k=\sqrt{m}$ is used). This parameter for waves in nature is not known in advance but can be found from the following transcendental equations

$$
U=\frac{H L^{2}}{h^{3}}=\frac{g H T^{2}}{h^{2}}=\frac{16}{3} m K^{2}(m),
$$

provided the wavelength (or period) and height, and the water depth are known. As it is typical for long waves, horizontal velocity in a cnoidal wave is independent of the depth and the vertical velocity (which is small as compared to the horizontal velocity) is a linear function of the position of the water particles.

As different from sine waves, the shape of a cnoidal wave is not universal. It depends on the parameter $m$, or equivalently on the wavelength, wave height and the local water depth (Fig. 1b). The limit $m \rightarrow 1$ corresponds to KdV solitons. Cnoidal waves resemble sine waves to some extent for $0 \leq m<1$. The limits $H \rightarrow 0$ or $h \rightarrow \infty$ correspond to $m \rightarrow 0$ and lead to pure sine waves. 

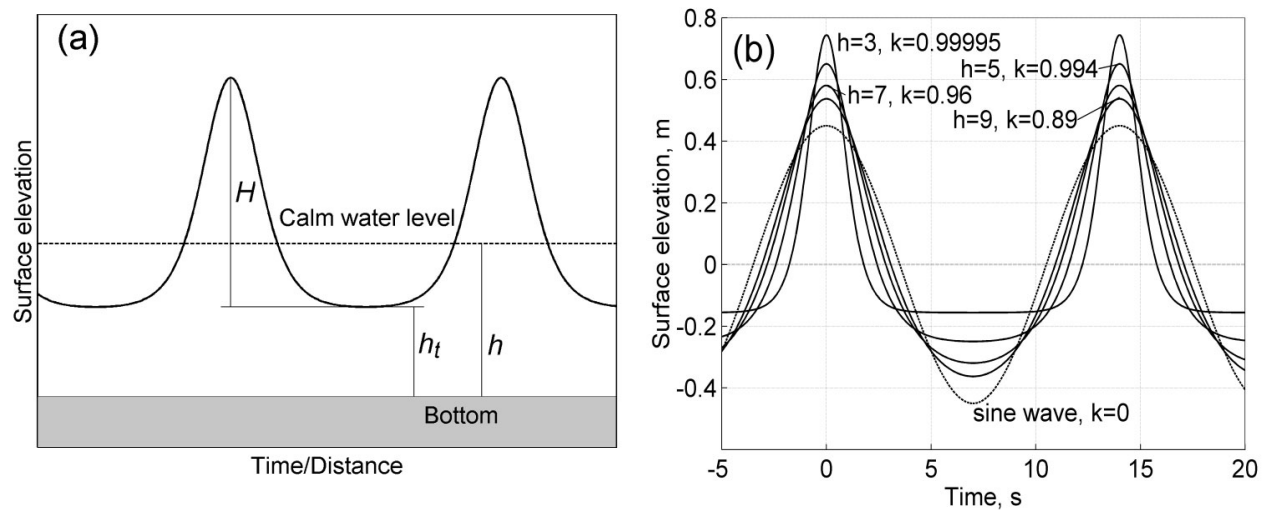

Fig. 1. (a) Definition sketch of a cnoidal wave; (b) comparison of profiles of a sine wave with the period of $14 \mathrm{~s}$ and height of $0.9 \mathrm{~m}$ with cnoidal waves for water depths of 3-9 m.

\section{FIELD EXPERIMENTS}

Ship wave parameters are usually measured with a pressure sensor $\left[{ }^{6,14}\right]$. This approach properly resolves wave period and height but often fails to describe the wave profile correctly. In order to catch relatively short wave components, the pressure sensor normally is mounted in the subsurface layer with the use of an anchor, rope and buoyancy $\left[{ }^{14,34}\right]$, and may be easily displaced by water motion in the horizontal direction. Wave-induced movements of the sensor may distort the details of the wave profile.

A small number of more elaborated techniques such as capacitance wave staff $\left[{ }^{10}\right]$, acoustic Doppler devices $\left[{ }^{35}\right]$, waveriding buoys $\left[{ }^{36}\right]$, and video recording of the water surface along a scale $\left[{ }^{37}\right]$ have been used in certain studies. They are capable of providing reliable wave profiles; however, to the knowledge of the authors, no systematic study of experimentally measured wave shapes is available in international publications.

In summer 2003, a series of experiments was carried out in a shallow area near Aegna jetty, Tallinn Bay, in the vicinity of a ship wave measurement site described in $\left[{ }^{14}\right]$ (Fig. 2). The goal was to establish the factual shape of long ship waves in shallow coastal areas. A pillar was rigidly fixed to the bottom in a vertical position so that its top reached out of water about $1 \mathrm{~m}$ above the calm sea level. It was slender enough $(\varnothing 50 \mathrm{~mm})$ not to create considerable reflected waves, and was stabilized with the use of three ropes. The fluctuations of the water surface along a scale on the pillar were recorded on the videotape. The resolution of the scale allowed detecting the position of the water surface with an accuracy of $1-2 \mathrm{~cm}$.

Measurements were performed in calm days in order to diminish the possible influence of wind waves. The behaviour of the water surface during about 2-3 hours at each site on three different days (July 14, 17 and 20) at four sites, with 


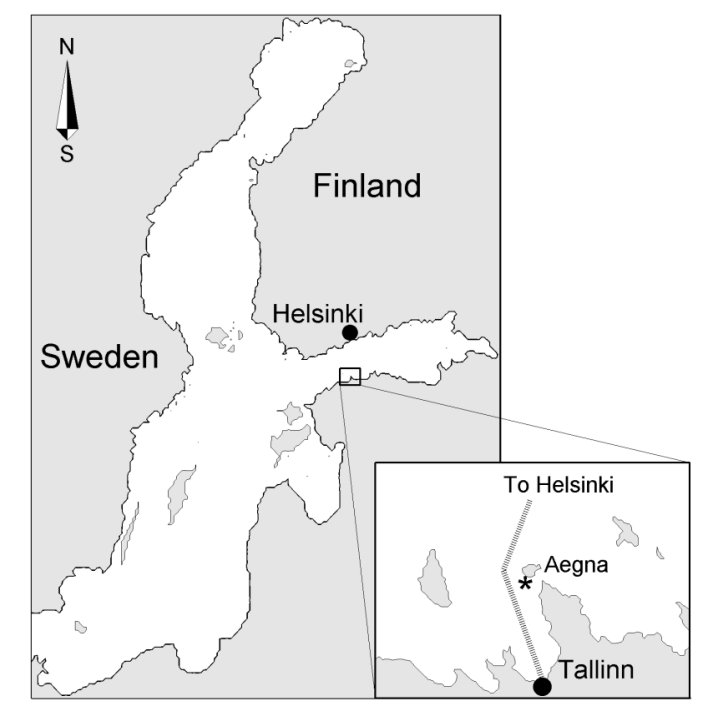

Fig. 2. Study area. The dotted line in the small map shows the Tallinn-Helsinki ship lane. The star indicates the measurement site.

the water depths of 3.6 and $4.4 \mathrm{~m}$, was recorded. At deeper sites, video recording was performed from a small boat, and wave troughs were partly invisible.

For the further analysis only segments, containing full profile of single waves with heights at least $0.1 \mathrm{~m}$, are used (Table 1). They all were recorded in the morning of July 20 at a measurement site about $30 \mathrm{~m}$ in the SSW direction from Aegna jetty where the water depth was $3.6 \mathrm{~m}$. Their total length is about $16 \mathrm{~min}$ and they represent 3 different wakes from ships sailing from Tallinn to Helsinki. Segments 2 and 3 actually represent different parts of the same wake. Owing to the malfunction of the timer of the video camera, the recorded wakes cannot be related to particular ships.

The water level in the frames was located and typed in manually. The changes of the water level are slow as compared to the frequency of recorded frames and only each 5th frame was digitized. The resulting time series show the temporal behaviour of the water surface with a resolution of $0.2 \mathrm{~s}$ at a fixed point,

Table 1. Parameters of segments of recordings used in the analysis

\begin{tabular}{c|c|c|c}
\hline $\begin{array}{c}\text { Segment } \\
\text { No. }\end{array}$ & $\begin{array}{c}\text { Duration, } \\
\mathrm{s}\end{array}$ & $\begin{array}{c}\text { Total number of waves according } \\
\text { to the zero-downcrossing or zero- } \\
\text { upcrossing method }\end{array}$ & $\begin{array}{c}\text { Number of waves, } \\
\text { suitable for further } \\
\text { analysis }\end{array}$ \\
\hline 1 & 198 & 18 & 17 \\
2 & 251 & 25 & 12 \\
3 & 120 & 20 & 17 \\
4 & 300 & 33 & 32
\end{tabular}




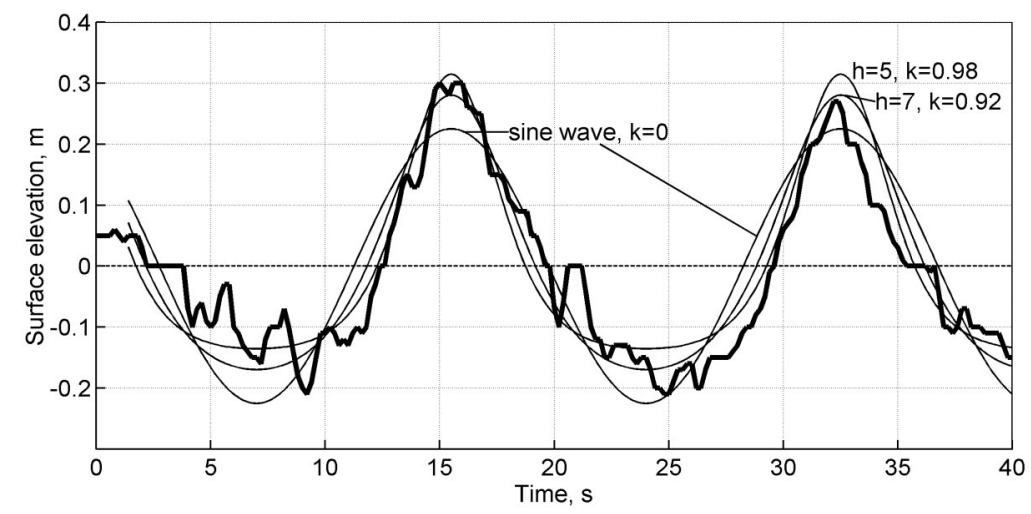

Fig. 3. Water surface (bold line) in ship waves with a height of about $0.5 \mathrm{~m}$ and a period of $16-17 \mathrm{~s}$ at a measurement site with a water depth of $3.6 \mathrm{~m}$, cnoidal waves for the water depths of 5 and $7 \mathrm{~m}$, and sine wave with the same parameters.

equivalently, the profile of the ship wake at this point. Even the shape of the highest recorded waves is generally regular and symmetrical with respect to their crests and throughs, thus the waves were far from breaking.

Figure 3 shows a part of the digitized record. It contains two subsequent crests of a ship wave with a height of about $45-50 \mathrm{~cm}$ and a period of about $17 \mathrm{~s}$. Small fluctuations of the water surface with a height of about $10 \mathrm{~cm}$ and a period of 1-2 $\mathrm{s}$ are wind waves. The two ship waves have modest heights (as compared with about $1 \mathrm{~m}$ high waves discussed in $\left[{ }^{14}\right]$ ), yet they considerably differ from a sine wave. Their crests are relatively narrow and rise much higher above the calm sea level compared with the dropdown of the water surface in the wave troughs.

\section{WAVE DATA}

An elliptical filter with $0.1 \mathrm{~dB}$ passband ripple, $60 \mathrm{~dB}$ stopband attenuation and $0.3 \mathrm{~Hz}$ cut-off was applied to the time series. Such a filtering removed the short wind wave background and smoothed out possible digitizing errors and small-scale wave reflections. As different from periodic wave trains, each wave in natural conditions has some individuality. This feature is the most important one in case of ship wakes that serve as ensembles of transient waves with largely varying properties $\left[{ }^{14}\right]$. For that reason, the analysis was performed for single waves, detected from the filtered water surface time series. The single waves, determined according to the zero-upcrossing and zero-downcrossing methods $\left[{ }^{38}\right]$, differ to some extent but the general features of both wave sets practically coincide. Below we present results, corresponding to the zero-downcrossing method. The total number of single waves is about 100 but the number of waves with a regular shape and with a height over $10 \mathrm{~cm}$, suitable for further analysis, is 78 . 
The wave period was defined as the time interval between subsequent down/upcrossings of the filtered water surface time series. The wave height was found from the non-filtered time series. The wavelength was calculated from the approximate dispersion relation $\omega=\kappa \sqrt{g h}$ for the depth of the measurement site.

Since the properties of ship-induced waves, remote from the ship lane, apparently are independent of the generation conditions (and are basically governed by the wave parameters), it is important that the measured data set matches waves of different height and period typically occurring in ship wakes. The height of the highest wake was about $0.95 \mathrm{~m}$, thus about $90 \%$ from the expected daily largest height of long waves at this site $\left[{ }^{14}\right]$. Other wakes had the highest waves of about 50 and $40 \mathrm{~cm}$. The mean height of the analysed waves was $0.26 \mathrm{~m}$. The period of the largest waves was $11-12 \mathrm{~s}$ whereas some waves had periods of 15-17 s and even a few waves with periods over $20 \mathrm{~s}$ occurred. Therefore, the recorded waves well represent the properties of ship waves at the measurement site $\left[{ }^{14}\right]$.

The applicability of different wave theories for ship-generated waves at the particular measurement site can be roughly estimated from the wave parameters. The ratio $L / h>10$ for most of the recorded waves, thus the Stokes theory (that requires $L / h<10$ ) may be incorrect for many ship-generated waves already with heights of about $0.2 \mathrm{~m}$ and is not applicable for ship waves higher than $0.4 \mathrm{~m}$ (Fig. 4a). In terms of the Ursell number, a non-linear theory must be applied if $U>1$. The cnoidal wave theory may be applied for waves with $U \geq 30$. An alternative theory for moderate Ursell numbers $(30 \leq U \leq 75)$ is the Stokes wave theory $\left[{ }^{18}\right]$. From Fig. $4 \mathrm{~b}$ it follows that the cnoidal wave theory is generally justified for recorded waves higher than $0.2 \mathrm{~m}$ and necessary for waves higher than $0.4-0.5 \mathrm{~m}$.
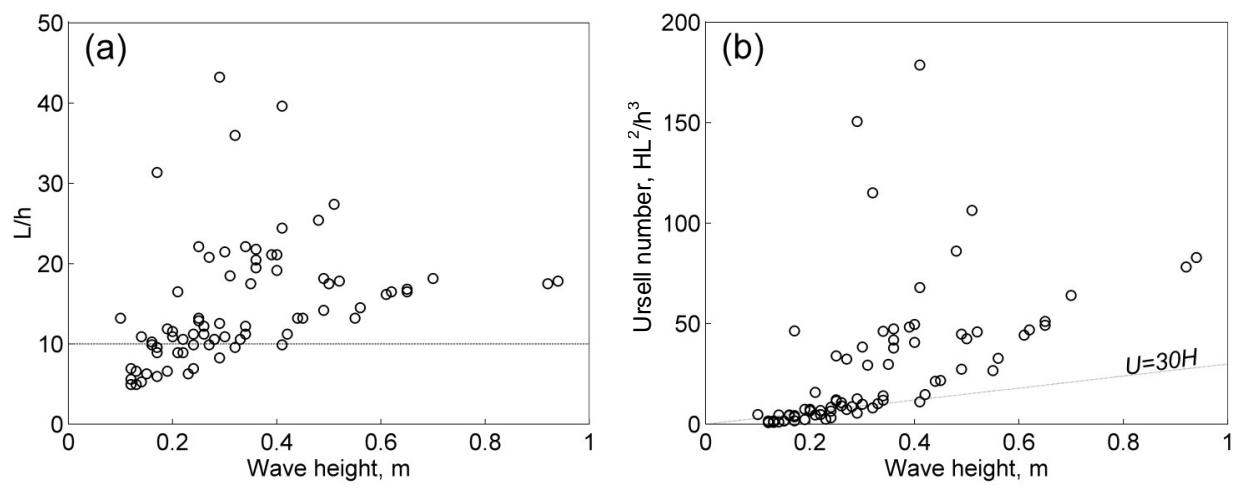

Fig. 4. (a) Scatter plot of the wave height and the ratio $L / h$ of the wavelength and the local water depth; the line $L / h=10$ indicates the approximate border of validity of the Stokes theory; (b) scatter plot of the wave height and the Ursell number. The line $U=30 H$ corresponds to waves with periods of about $6 \mathrm{~s}$. 
Within the set of measured waves, the Ursell number $U$ varies largely, from $U \leq 20$ for waves with $H \leq 0.2 \mathrm{~m}$ and $U \geq 50$ for several waves with a height of $0.3-0.4 \mathrm{~m}$ or higher (Fig. $4 \mathrm{~b}$ ). Roughly one half of the measured waves (the waves with heights $<0.4 \mathrm{~m}$; the corresponding bullets are concentrated along the line $U=30 \mathrm{H}$ ) have the length of about $40 \mathrm{~m}$ and the period of about $6 \mathrm{~s}$. It is interesting that Ursell numbers for waves with roughly equal heights $H \geq 0.3 \mathrm{~m}$ have a very large scatter. This feature means that waves with largely different lengths and periods but with comparable heights occur in ship wakes.

Each measured wave was demeaned and then compared with cnoidal waves with the same length and height but corresponding to different values of the parameter $m$, or equivalently, to different water depths. Doing so is justified by the fact that sea waves usually propagate over an inhomogeneous bottom and the depth at the single measurement point not necessarily represents the depth that controls the shape of the wave. The best fit (minimizing the root mean square deviation of pointwise comparison of the measured and the theoretical profiles) was sought for depths 1-30 m. Phase shifts of factual waves, possibly caused by the use of the filtered time series for selecting the single waves, were compensated, if necessary. Finally, the parameter $k=\sqrt{m}$ of the Jacobi elliptical integrals (Fig. 5) was determined from Eq. (4) for both the factual depth and for the depth of the best fit.

The depth, corresponding to the best fit, generally somewhat exceeds the water depth at the measurement site. This feature apparently reflects the fact that the waves were propagating onshore towards shallower areas whereas a large part of measured waves lied in a deeper area. However, the two depths insignificantly differed for most of the waves.
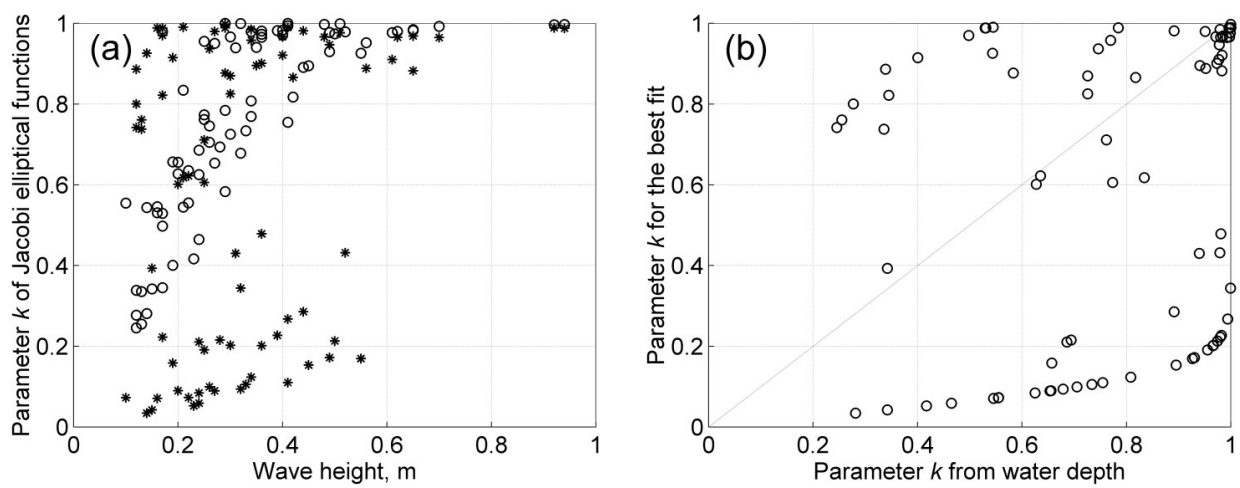

Fig. 5. (a) Scatter plot of the wave height and the parameter $k=\sqrt{m}$ of Jacobi elliptical functions (bullets - defined according to the factual measurement depth, stars - according to the best fit); (b) scatter plot of the parameter $k$, defined for the factual water depth versus this parameter corresponding to the depth of best fit. The alignment of bullets along a curve in the lower part of panel (b) is caused by limitations of the procedure for determining the best fit, according to which the maximum depth was set to $30 \mathrm{~m}$. 
The parameter $k_{\mathrm{d}}$ of Jacobi elliptical functions, computed from the factual depth, is moderate $\left(k_{\mathrm{d}} \leq 0.8\right)$ for wave heights not exceeding $0.2 \mathrm{~m}$ (Fig. 5a). It rapidly increases with the increase of wave heights, is $0.95-0.99$ also for several waves with heights of $0.2-0.4 \mathrm{~m}$ and is very close to 1 for waves with $H \geq 0.4 \mathrm{~m}$. The values of $k_{\mathrm{f}}$, corresponding to the depth of the best fit, are much more scattered. The corresponding points in Fig. 5a form two well distinguishable clusters. One cluster involves waves with $k_{\mathrm{f}} \geq 0.7$ that are evidently non-linear. Another cluster contains waves, characterized by $k_{\mathrm{f}} \leq 0.4$, that are close to linear waves. The clusters apparently correspond to the first and the second wave group of the ship wakes $\left[{ }^{6,14,29}\right]$.

Note that the waves with an equal height frequently correspond to drastically different values of $k_{\mathrm{d}}$ or $k_{\mathrm{f}}$ in Fig. 5a, therefore the wave height is not a complete measure of the shape or properties of ship-induced waves in the area in question. In the framework of both the Stokes and the cnoidal wave theory, the properties of wave trains in an area with a flat bottom are comprehensively described by the length and height of the waves, and the water depth. Thus, the wave shape and the corresponding Jacobi parameter should be identical for waves with the same height and length. A somewhat unexpected feature is that the parameters $k_{\mathrm{d}}$ and $k_{\mathrm{f}}$ for single waves frequently scatter drastically (Fig. 5b). Therefore the shape of a single ship wave in shallow areas is only weakly dependent on the wave parameters. This feature may be interpreted as a manifestation of non-linear interactions in groups of cnoidal waves that becomes evident in the form of spatio-temporal phase shifts of single waves $\left[{ }^{39}\right]$ and may lead to considerable distortion of wave profiles.

However, there exists a set of waves for which $k_{\mathrm{d}} \approx k_{\mathrm{f}} \approx 0.9-0.999$ that includes all waves with the height over $0.5 \mathrm{~m}$ (Fig. 5a). Their shapes greatly differ from that of sine waves and are very close to the shape of KdV solitons. Certainly, a water surface record at a single point does not prove that these waves are solitons, because it is not clear whether they maintain their shape in time and interact elastically with each other. Yet it serves as indirect evidence that ship wakes may form a sequence of solitons in shallow areas.

\section{APPROXIMATIONS WITH CNOIDAL AND SINE PROFILES}

The classical wave theory (that is equivalent to cnoidal wave theory with $m=0$ ) reasonably reproduces both the elevation (Fig. 6a) and dropdown (Fig. 6b), caused by relatively small $(H \leq 0.3 \mathrm{~m})$ ship waves. The elevation and dropdown in such waves are practically symmetrical with respect to the calm water level. Higher waves are often asymmetrical. Figure 6 confirms that the cnoidal wave theory provides a reasonable fit of their shape and associated behaviour of the water surface. However, the classical wave theory systematically considerably underestimates the maximum elevation at wave crests and overestimates the dropdown at wave troughs for waves with an amplitude exceeding $0.15 \mathrm{~m}(H>0.3 \mathrm{~m})$. 

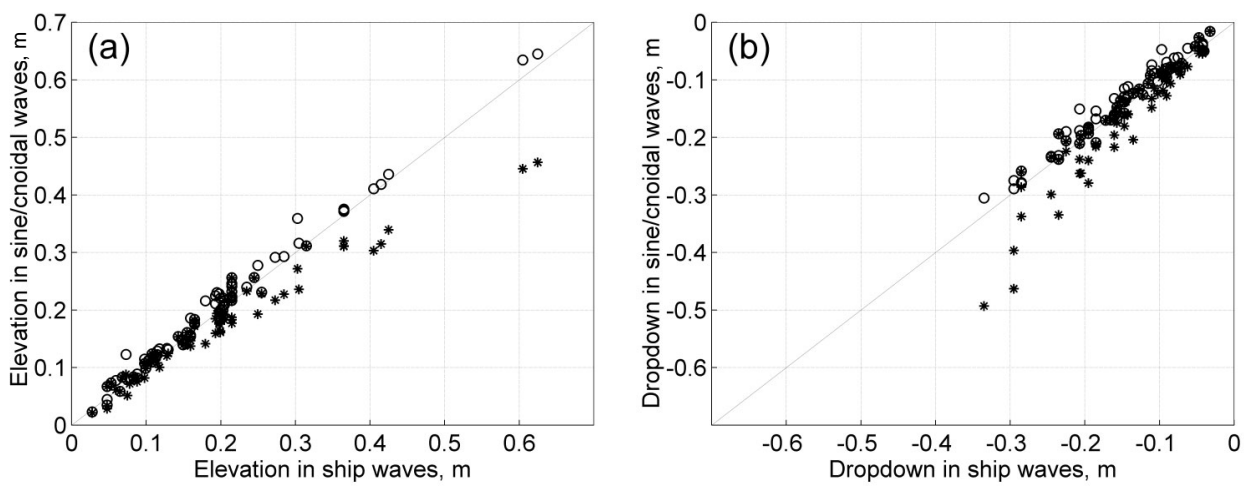

Fig. 6. Maximum water elevation (a) and dropdown (b) caused by sine waves (stars) and cnoidal waves (according to the depth of the best fit, bullets) versus the recorded elevation and dropdown in ship waves. The data are given with respect to instantaneous mean water level in single waves with the use of non-filtered water surface time series.

The cnoidal theory seems to overestimate slightly the maximum elevation and dropdown; however, the relative error is several percents. The relative error of the classical wave theory for the maximum elevation or dropdown is roughly $20 \%$ for waves with an amplitude of $0.2-0.3 \mathrm{~m}(H=0.4-0.6 \mathrm{~m})$ and reaches about $40 \%$ (absolute error $0.2 \mathrm{~m}$ ) for the highest recorded examples of ship waves.

Comparison of several ship waves with cnoidal and sine waves (Fig. 7) shows that, as expected, the cnoidal waves form a nearly perfect fit for the recorded waves. Sine waves more or less correctly follow the crests of relatively small waves with a height of about $0.4 \mathrm{~m}$. For larger waves, they overestimate the water dropdown and underestimate the elevation.

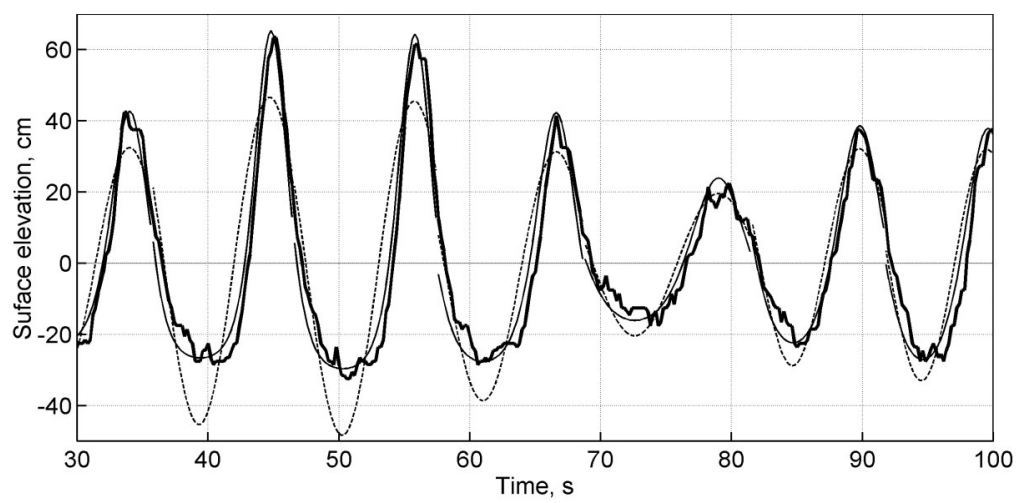

Fig. 7. Water surface time series (bold line) in a ship wake with a maximum wave height of $0.95 \mathrm{~m}$, the shape of cnoidal waves corresponding to the depth of the best fit (solid line), and the corresponding sine wave (dashed line). 

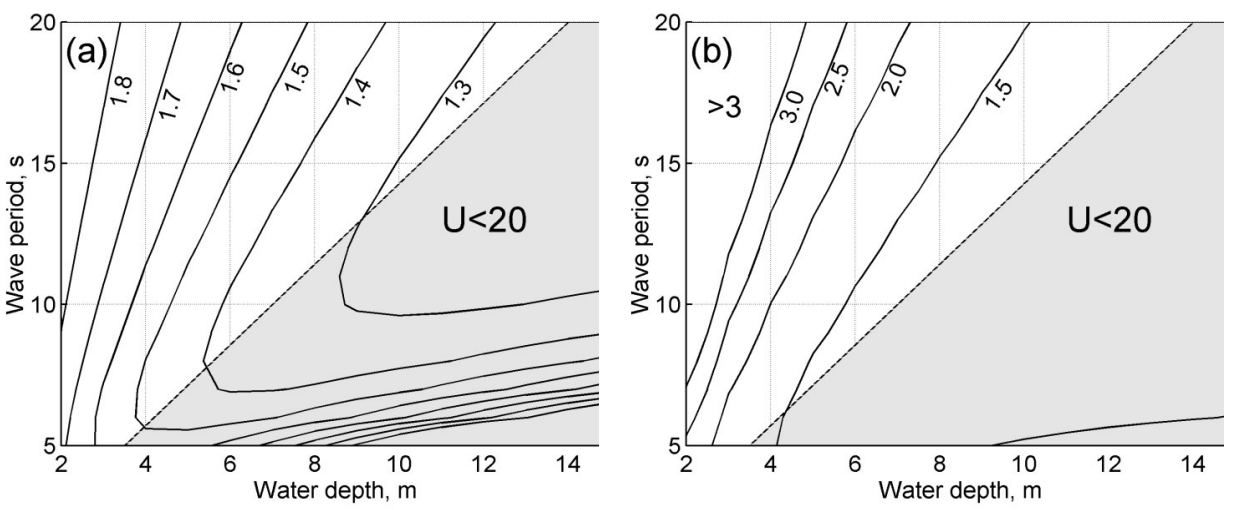

Fig. 8. (a) Ratio of maximum near-bottom velocity, induced by cnoidal and sine waves for the wave height of $1 \mathrm{~m}$; (b) ratio of maximum vertical velocities, induced by cnoidal and sine waves at the half depth of the water column. In the area, where $U<20$, the cnoidal wave theory is not preferable.

The crest of a cnoidal wave is narrower and its trough is more flat compared with a sine wave of the same height and length (Fig. 1a). Since the velocity components follow the surface displacement in long waves, the maximum speed of water particles in cnoidal waves apparently exceeds that in sine waves.

The ratio of maximum velocities of water particles in cnoidal and sine waves with periods of 5-20 s and a height of $1 \mathrm{~m}$ (based on Eqs. (1)-(3)) is presented in Fig. 8. The maximum velocity of water particles in cnoidal waves greatly exceeds that in sine waves for all the combinations of wave parameters for which the cnoidal theory is applicable. The smaller the water depth or the larger the wave, the larger is the difference. The near-bottom velocity in cnoidal waves remarkably (about 1.5 times) exceeds that in sine waves for waves with periods of $15 \mathrm{~s}$ already in $6 \mathrm{~m}$ deep water and markedly (about 30\%) for such waves in about $10 \mathrm{~m}$ deep water. This difference does not imply larger energy of cnoidal waves, because high speeds occur in a small part of the wave. The difference of vertical velocity is even larger, but the absolute values of this velocity component are by an order of magnitude smaller than the near-bottom velocity.

\section{CONCLUSIONS AND DISCUSSION}

Although the shape and properties of long waves in shallow areas with flat bottom should exhibit non-linear features and resemble those of cnoidal waves for certain combinations of wave parameters and the water depth, the sea bottom is not perfect and may greatly affect the actual wave shape. The performed experiments demonstrate that the shape of water surface in a large part of ship-generated waves, reaching the coastal area of Tallinn Bay, is well described by the cnoidal wave theory. The linear wave theory frequently fails to match the wave profile. Ship 
waves of already relatively small height (about $0.4 \mathrm{~m}$ ) considerably differ from sine waves at a depth of about $4 \mathrm{~m}$. For wind waves of comparable height the linear theory is yet applicable at this depth, because they are much shorter than ship waves $\left[{ }^{14,15}\right]$.

Although there exist various descriptions of weakly non-linear surface waves, in the current paper we have used only the cnoidal wave theory. The reason is that the shape of a cnoidal wave has relatively simple analytical expression. This feature allows fast comparison of the measured data with theoretical predictions whereas, for example, description of the Boussinesq-type waves contain quite complicated expressions $\left[{ }^{18}\right]$.

The parameter $k$ in the Jacobi elliptical functions is very close to 1 for many waves with a shape practically coinciding with that of the solitary wave solution of the KdV equation. These waves not necessarily are solitons, because not only the instantaneous shape but preserving it in time and in collisions is the distinguishing feature of solitons. Yet cnoidal waves of relatively large amplitude preserve their identity and shape fairly well in time, and partially also during interactions $\left[{ }^{40}\right]$. Therefore it is likely that dynamics of a certain part of ship wakes in shallow areas is very close to that of the ensembles of $\mathrm{KdV}$ solitons.

Since water motion in long surface waves mainly follows their shape, the results obtained in this study suggest that the classical wave theory frequently fails to correctly forecast the temporal behaviour and the maximum values of the velocity field in ship waves. The analysis above suggests that velocities of water particles in long ship waves apparently are much larger than expected in the classical approach. A large difference may frequently occur in areas where the non-linear wave theory is preferable, i.e., for depths less than 10-15 m, depending on the wave period and height.

The propagation of ship waves generally is estimated based either directly or indirectly (for example, with the use of spectral models) on the linear wave theory $\left[{ }^{10,13}\right]$. Only a few applications of non-linear theories in this area make use of basic properties of non-linear waves such as their excessive phase speed $\left[{ }^{29}\right]$. Such models reasonably represent the overall behaviour of the ship wash and the height and length of its components in shallow areas.

An important outcome from the performed studies is that properties of single waves from each ship wake have a large scatter. The shape of a part of waves is very similar to highly cnoidal waves whereas other waves of comparable height and length from the same wake are practically sinusoidal. The general tendency is that the leading (that is, the longest and the highest) waves are closer to cnoidal waves than shorter wake components.

Since many properties of long waves, in particular, velocities of water particles, follow the shape of the waves, at least a part of ship waves exhibits nonlinear features. Therefore, an appropriate non-linear theory for properties of wave-induced motions should be used in shallow areas (in particular, in estimates of the maximum near-bottom velocities in long ship waves). 
Owing to their non-linear properties, the influence of a part of long ship waves on seabed, offshore structures and local ecosystem in certain parts of the coastal slope apparently is much larger than expected from the linear wave theory. Since ship waves are a new component of the water dynamics in many areas, an extensive reaction of the benthic layer and fine bottom sediments to the frequent occurrence of non-linear waves is likely in the affected regions. Although species that prefer rocky or sandy bottom may benefit from the increased hydrodynamic activity, the concern is that abrupt changes in forcing conditions usually have an adverse effect on the local ecosystem. The reduced water transparency, an apparent feature of such reaction $\left[{ }^{9,12}\right]$, may have strong suppressing feedback on the bottom vegetation, and suspension and re-sedimentation of finer sediments may considerably worsen fish spawning conditions.

The specific features of ship waves become evident when they are much longer than natural waves. The results of the study are therefore applicable in micro-tidal basins that are sheltered from long ocean swell and that have extensive shallow coastal areas (for instance, lakes hosting fast ferry traffic, the Azov Sea, the north-western part of the Black Sea, and certain parts of the Mediterranean).

Both the length and height of waves generally increase with the ship speed $\left[{ }^{6,13,41}\right]$. Therefore, environmental impact of fast ferry traffic eventually may be reduced by means of reducing speed of certain types of ships. Starting from 1997, several countries and communities have introduced regulations (primarily based on the wave height criteria in the nearshore environment $\left[{ }^{10}\right]$ ) for the fast ferry traffic. Relevant restrictions for Tallinn Bay have been discussed many times $\left[{ }^{8}\right]$ but no decision has been made yet.

\section{ACKNOWLEDGEMENTS}

This study has been supported by the Estonian Science Foundation (grant No. 5762) and by the Centre for Nonlinear Studies (CENS) at the Institute of Cybernetics, Tallinn University of Technology.

\section{REFERENCES}

1. Nanson, G. C., von Krusenstierna, A. and Bryant, E. A. Experimental measurements of riverbank erosion caused by boat-generated waves on the Gordon River, Tasmania. Regul. Rivers Res. Manage., 1994, 9, 1-14.

2. Gaskin, S. J., Pieterse, J., Al Shafie, A. and Lepage, S. Erosion of undisturbed clay samples from the banks of the St. Lawrence River. Can. J. Civil Eng., 2003, 30, 585-595.

3. Bourne, J. Louisiana's vanishing wetlands: going, going... Science, 2000, 289, 1860-1863.

4. Ali, M. M., Murphy, K. J. and Langendorff, J. Interrelations of river ship traffic with aquatic plants in the River Nile, Upper Egypt. Hydrobiol., 1999, 415, 93-100.

5. Lindholm, T., Svartström, M., Spoof, L. and Meriluoto, J. Effects of ship traffic on archipelago waters off the Långnäs harbour in Åland, SW Finland. Hydrobiol., 2001, 444, 217-225.

6. Guidelines for Managing Wake Wash from High-Speed Vessels. Report of the Working Group 41 of the Maritime Navigation Commission. International Navigation Association (PIANC), Brussels, 2003. 
7. Fast Ferries as a Qualitatively New Key Forcing Factor of Environmental Processes. MEPC 51/21, 20.01.2004, Marine Environment Protection Committee, International Maritime Organisation, London, 2004.

8. Soomere, T., Elken, J., Kask, J., Keevallik, S., Kõuts, T., Metsaveer, J. and Peterson, P. Fast ferries as a new key forcing factor in Tallinn Bay. Proc. Estonian Acad. Sci. Eng., 2003, 9, 220-242.

9. Osborne, P. D. and Boak, E. H. Sediment suspension and morphological response under vesselgenerated wave groups: Torpedo Bay, Auckland, New Zealand. J. Coastal Res., 1999, 15, 388-398.

10. Parnell, K. E. and Kofoed-Hansen, H. Wakes from large high-speed ferries in confined coastal waters: management approaches with examples from New Zealand and Denmark, Coastal Manage., 2001, 29, 217-237.

11. Soomere, T. and Kask, J. A specific impact of waves of fast ferries on sediment transport processes of Tallinn Bay. Proc. Estonian Acad. Sci. Biol. Ecol., 2003, 52, 319-331.

12. Erm, A. and Soomere, T. Influence of fast ship waves on optical properties of sea water in Tallinn Bay, Baltic Sea. Proc. Estonian Acad. Sci. Biol. Ecol., 2004, 53, 161-178.

13. Kirk McClure Morton. Investigation of High Speed Craft on Routes Near to Land or Enclosed Estuaries. Research Rep. JR226. The Maritime and Coastguard Agency, UK, 1998.

14. Soomere, T. and Rannat, K. An experimental study of wind waves and ship wakes in Tallinn Bay. Proc. Estonian Acad. Sci. Eng., 2003, 9, 157-184.

15. Soomere, T. Wind wave statistics in Tallinn Bay. Boreal Environ. Res., 2005, 10, 103-118.

16. Coates, T. T. and Hawkes, P. J. Beach recharge design and bi-modal wave spectra. In Coastal Engineering 1998: Proc. 26th International Conference. Copenhagen, 1998 (Edge, B. L., ed.). American Society of Civil Engineers, 1999, vol. 3, 3036-3045.

17. Hawkes, P. J. Mean overtopping rate in swell and bimodal seas. Proc. Inst. Civil Eng. Water Marit. Energy, 1999, 136, 235-238.

18. Massel, S. R. Hydrodynamics of Coastal Zones. Elsevier, Amsterdam, 1989.

19. Gourlay, T. P. The supercritical bore produced by a high-speed ship in a channel. J. Fluid Mech., 2001, 434, 399-409.

20. Katsis, C. and Akylas, T. R. On the excitation of long nonlinear water waves by a moving pressure distribution. Part 2. Three-dimensional effects. J. Fluid Mech., 1987, 177, 49-65.

21. Li, Y. and Sclavounos, P. D. Three-dimensional nonlinear solitary waves in shallow water generated by an advancing disturbance. J. Fluid Mech., 2002, 470, 383-410.

22. Lee, S. J. and Grimshaw, R. H. J. Upstream-advancing waves generated by three-dimensional moving disturbances. Phys. Fluids A, 1990, 2, 194-201.

23. Jiang, T. Ship Waves in Shallow Water. Fortschritt-Berichte VDI, Reihe 12, Nr. 466, VDI Verlag, Düsseldorf, 2001.

24. Chen, X.-N. and Sharma, S. D. A slender ship moving at a near-critical speed in a shallow channel. J. Fluid Mech., 1995, 291, 263-285.

25. Jiang, T. Investigation of waves generated by ships in shallow water. In 22nd Symposium on Naval Hydrodynamics. Washington DC, 1998. National Academy Press, Washington DC, 1998, 601-612.

26. Jiang, T., Henn, R. and Sharma, S. D. Wash wave generated by ships moving on fairways of varying topography. In 22nd Symposium on Naval Hydrodynamics. Fukuoka, 2002. National Academy Press, Washington DC, 2003, 441-457 (www.nap.edu).

27. Kofoed-Hansen, H., Jensen, T., Sørensen, O. R. and Fuchs, J. Wake wash risk assessment of high-speed ferry routes - a case study and suggestions for model improvements. In Proc. International Conference on Hydrodynamics of High Speed Craft, Wake Wash and Motion Control. London, 2000. The Royal Institute of Naval Architects; also Report No. 2000/52, Danish Hydraulic Institute, Copenhagen, 2000.

28. Kofoed-Hansen, H. Technical Investigation of Wake Wave from Fast Ferries. Report No. 965012, Danish Hydraulic Institute, Copenhagen, 1996. 
29. Whittaker, T. J. T., Doyle, R. and Elsäßer, B. A study of the leading long period waves in fast ferry wash. In Proc. International Conference Hydrodynamics of High Speed Craft - Wake Wash and Motions Control. London, 2000. RINA, London, 2000, paper 7 (CD).

30. Neuman, D. G., Tapio, E., Haggard, D., Laws, K. E. and Bland, R. W. Observation of long waves generated by ferries, Can. J. Remote Sens., 2001, 27, 361-370.

31. Hammack, J., McCallister, D., Scheffner, N. and Segur, H. Two-dimensional periodic waves in shallow water. Part 2. Asymmetric waves. J. Fluid Mech., 1995, 285, 95-122.

32. Peterson, P., Soomere, T., Engelbrecht, J. and van Groesen, E. Soliton interaction as a possible model for extreme waves in shallow water. Nonlinear Proc. Geophys., 2003, 10, 503-510.

33. Soomere, T. and Engelbrecht, J. Extreme elevations and slopes of interacting solitons in shallow water. Wave Motion, 2005, 41, 179-192.

34. Stumbo, S., Fox, K., Dvorak, F. and Elliot, L. The prediction, measurement, and analysis of wake wash from marine vessels. Mar. Technol. SNAME News, 1999, 36, 248-260.

35. Fissel, D., Billeness, D., Lemon, D. and Readshaw, J. Measurement of the wave wash generated by fast ferries with upward looking sonar instrumentation. In Proc. 17th International Fast Ferry Conference. New Orleans, USA, 2001 (CD).

36. Koushan, K., Werenskiold, P., Zhao, R. and Lawless, J. Experimental and theoretical investigation of wake wash. In Proc. 6th International Conference on Fast Sea Transportation FAST 2001. Southampton, 2001. RINA, London, 2001, vol. 1, 165-179.

37. Hannon, M. and Varyani, K. S. The wash effect of high speed ferries in coastal and inland waterways. In Proc. International Conference on Coastal Ships and Inland Waterways. London, 1999. RINA, London, 1999 (CD).

38. IAHR working group on wave generation and analysis. List of sea-state parameters. J. Waterw. Port Coast. Ocean Eng., 1989, 115, 793-808.

39. Osborne, A. R., Serio, M., Bergamasco, L. and Cavaleri, L. Solitons, cnoidal waves and nonlinear interactions in shallow-water ocean surface waves. Physica D, 1998, 123, 64-81.

40. Svendsen, I. A. and Veeramony, J. Wave breaking in wave groups. J. Waterw. Port Coast. Ocean Eng., 2001, 127, 200-212.

41. Sorensen, R. M. Ship-generated waves. Adv. Hydrosci., 1973, 9, 49-83.

\section{Kiirlaevalainete kuju Tallinna lahe rannavööndis}

\section{Tarmo Soomere, Reio Põder, Kalev Rannat ja Andres Kask}

Veetaseme salvestuste alusel on analüüsitud pikkade laevalainete kuju ja omadusi Tallinna lahe madalaveelises osas. Mittelineaarsed efektid modifitseerivad neid laineid alates sügavustest $10-15 \mathrm{~m}$. Sügavustes alla $4-5 \mathrm{~m}$ sarnanevad laevalained kõrgusega üle 0,4 m knoidaalsete lainetega, kusjuures kõrgeimatel lainetel on Korteweg-de Vriesi solitonide kuju. Sama pikkade ja kõrgete siinuslainetega võrreldes põhjustavad sellised lained oluliselt suuremaid vee kiirusi nii põhja lähedal kui ka veesambas tervikuna. 